Mia Consalvo

\title{
Rule Sets, Cheating, and Magic Circles: Studying Games and Ethics
}

\begin{abstract}
:
This paper provides frameworks for understanding how ethics might be expressed in gameplay situations, and how we can study the ethical frameworks that games offer to players. There are many ways to delve into such topics, and this paper considers only a few approaches. It briefly surveys some of the important questions and critiques arising from audience studies, theories of play and games, and work on cheating, and begins to build a framework for considering ethics in relation to games and players that transcends the "place apart" that games are often constructed as.
\end{abstract}

\section{Agenda}

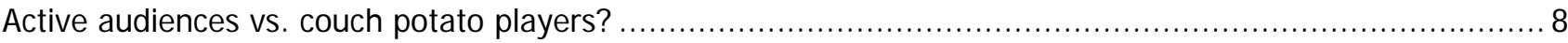

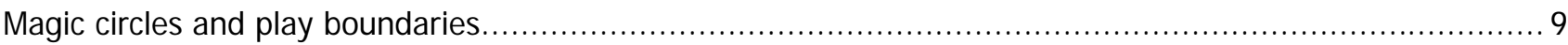

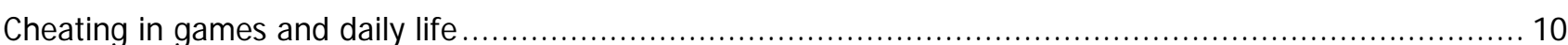

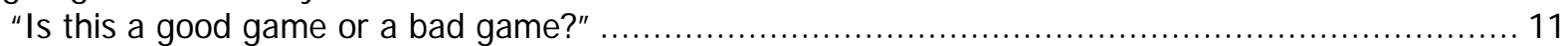

\section{Author:}

Mia Consalvo:

- Organization and contact address: 213 RTV Building, School of Telecommunications, 9 South College Street, Ohio University, Athens, Ohio 45701, USA

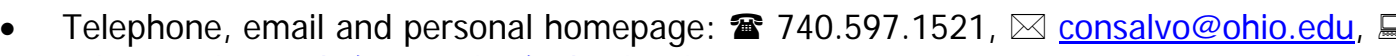
oak. cats. ohiou. edu/ consalvo/index.html

- Relevant publications:

- Console video games and global corporations: Creating a hybrid culture, New Media \& Society, forthcoming.

- Cheating can be good for you: Educational games and multiple play styles, On the Horizon, 13 (2), July 2005. 
For the past four years I've been asking game players how they define cheating in games, and how they negotiate and enact cheating practices. Some react as if I'm asking them to reveal their utter lack of ethics and values, and they then respond with clear denunciations that cheating is wrong and they would "never do anything like that." When asked "like what," answers begin to fragment, and lose moral certainty. Clearly, we need a better understanding of how ethics might be expressed in gameplay situations, and how we can study the ethical frameworks that games offer to players. Research in this area is beginning (Reynolds, 2002), but many interesting questions remain to be asked.

There are many ways to delve into such topics, and this paper considers only a few approaches. It briefly surveys some of the important questions and critiques arising from audience studies, theories of play and games, and work on cheating, and begins to build a framework for considering ethics in relation to games and players that transcends the "place apart" that games are often constructed as.

\section{Active audiences vs. couch potato players?}

Much of the earliest research focusing on game players took a social scientific approach, seeking to learn how violence in games (the central concern) "affected" various types of players, in different ways (Sherry, 2001). Although critiqued by many (Goldstein, 2005; Jenkins, 1999), that paradigm has continued to shape how many individuals view the playing experience. Gamers are often seen as actively participating in games, but at the same time being actively (and negatively) affected by the content within the game. Fundamental to such an approach is the negation (or dismissal) of how players constantly make choices about their in-game behavior, as well as consider and frame their game actions relative to other daily situations and contexts. While it has not been much linked to gameplay yet, active audience theory can aid us in better understanding player choices and decisions, and the ways that individuals negotiate meanings drawn from a wide variety of "texts."

Theorization of an active media audience has a strong history, with current questions investigating the degree to which resistance and negotiation might take place in viewing situations, rather than debate if such activities occur at all (Fiske, 1987). The conceptualization of the active audience arose to counter effects' theorists insistence on passive media consumers sitting at the end of a one way tunnel of content-the receiver that might contend with some noise but ultimately was supposed to take delivery of an intended message. Media theorists have accepted ideas of active audiences, yet the term "active" is always attached, perhaps to remind ourselves that the term "audience" implies someone not as active in the process as we would hope. Active audience theory also confirms the presence of polysemic content, which challenges the view that media texts are closed, or can be read or understood in one particular way. Hall has carefully explored the encoding process, arguing that even as media producers attempt to control the meanings they embed in their messages, that process is always incomplete or partial (Hall, 1973/1980).

Considering the interactive nature of videogames, polysemic content as a conceptual category with which to think about games becomes even more relevant. While television shows and films may invite different interpretations, the viewer is confronting a somewhat static text, with an unchanging story or plotline. Indeed, some audience theorists have contested the idea that viewers easily or radically reinterpret texts, as such processes take energy and often result in little pleasure (Kellner, 2005). However, games are hardly static-they are as Aarseth argues, ergodic texts, requiring non-trivial effort to explore. Games also have varying levels of interactivity, which demands that players make choices, choices which can then alter (sometimes very distinctly) the story or experience of a particular game.

Games such as those in the Grand Theft Auto series perfectly illustrate the conditions and consequences created by polysemic content. Players are given the opportunity to follow the game designer's storyline(s) involving mission completion, as well as chances to explore the space of the gameworld, which is almost completely interactive. More and less violent solutions to problems can often be found, and players can create their own "versions" of the game. Likewise, the PC game The Sims has no pre-set storyline, allowing players to explore all sorts of "what if" ideas they may imagine for their simulated people.

Game playing by definition (and in practice) demands activity and volition. How could a person passively play a game? The player is fundamentally implicated in the gameplay situation, and referring back to Hall (1973/1980) and Fiske (1987), we see that players are constantly making meanings, decoding icons or actions or texts in the game. And 
different players of varying ages, social classes, nationalities, ethnicities and genders bring their own experiences with them to each game. They may be hardcore or casual (whatever those terms imply). They may be dedicated Role-Playing gamers but trying their first Real-Time Strategy game (RTS). Or they may be replaying their favorite games for nostalgia and enjoyment. Given such variables, and such activity, it seems almost ludicrous to posit an "effect" of one game on particular players or one game experience for all players. Better to wonder: How do individuals make games part of their lives? What do they make of the gameplay experience?

That starts us off in a more productive direction. We can look at players and games and their intersection to ask: Do games pose interesting ethical questions for players to take up? What layers or levels are involved? For example, many games offer the player the opportunity to revert to a previously saved version of the game. So if I feel guilty about leaving my Sim zombie fenced up outside to die (which I did), I can revert back to a stage of the game where he's still alive (which I didn't). How do players think about and engage with such choices? Are players seeing such opportunities in games to experiment with ethical decision-making? Is Sim "murder" a common activity? What reasons do players construct for such actions? Furthermore, how has our larger culture(s) portrayed games and what implications does that picture have for how we all approach (and judge) games?

So what is game ethics or what would it look like? To begin, there are at least several layers that we can consider as a basis for asking questions. The actions and choices made and offered by game developers, game publishers, marketers, game players, and the choices coded into the game itself can all be investigated. Here are just a few examples of where such questioning can lead.

In the game industry, for example, we can look at the decisions made by a company such as Rockstar Games, developer of a string of controversial titles such as the Grand Theft Auto series, Manhunt, and the forthcoming The Warriors. What did the company's management consider when deciding to create such games? Was the potential for controversy and divisiveness considered? Was it considered a positive or negative component of the each game's release? Does the company have any wider responsibility to the game development community? Do their games set precedents for legislation? Should the actions of one game company speak for all game companies?
Moving down a level, we can ask what game developers consider as they build games. How much violence and of what type is considered acceptable? Does that change with different player demographics? Do game developers even see their software coding in ethical ways? What about the design of individual characters-both central and peripheral avatars?

Finally, we can examine the individual player. How do players make choices about what they will or won't do in games? Do they follow rules in all circumstances or bend rules to achieve a greater good? Would a player shoot a dog in a game if that was the only way to win? How does a player justify murder in a game? Do players position the experience as "just a game" or as a cathartic release from everyday pressures?

Such questions only scratch the surface of what we can investigate in relation to games and ethics. Yet they point to central issues and areas of interest. We need to move beyond the simplistic ideas of good and bad, legal and illegal, to the more interesting and relevant factors related to the process of making moral choices. How do developers, publishers and players decide what is right and wrong? What do they conclude is right and wrong for them? And how does that play into or break through a magic circle into the everyday? We're only beginning to ask such questions-the answers should prove fascinating.

\section{Magic circles and play boundaries}

Another critical area to consider is the role of games and play in our lives, and how the spaces of games intersect with the spaces of daily life. Huizinga felt that play and games were central experiences of human beings, and went so far as to argue that play constituted culture (1955). While games have always existed, they defy easy categorization-as games can be for fun or in deadly earnest (as in war games), with no stakes or high stakes involved. Games can involve escape, but not always. Huizinga felt that games were protected by a "magic circle" or bounded space set apart from the everyday (much like the difference between the sacred and the profane), with rules as a boundary system for maintaining them.

Yet is this indeed true, or a useful way to think of games? Is there some boundary that delimits the playing field, separating the game from other, nongame space? If we take this idea to be valid, what 
happens to our conceptions about games? In that scenario, games are walled off as a space apartwhere we can create different rules, rewards, and punishments for the activities that take place within. Killing can be rewarded, and civilizations might best be taken over by "culture flipping" them. Players can experiment (to greater and lesser degrees) with potential actions, including exploring, socializing, empathizing, killing, being selfish, being silly, being inconsistent, or being all powerful. The results of those actions will vary based on the game being played, and its own particular rule set. Attempts to "game the game" can also provide players with elaborate, rich opportunities for exploration, experimentation and greater knowledge.

If we acknowledge games can provide such opportunities in "walled off" spaces, is it appropriate to judge games, or game player actions, by an external set of rules-rules that originate outside the magic circle? Games may reward players for particular actions-actions that would definitely not be rewarded in daily life. But should our standards for appropriate actions in daily life carry over to our game life? The Sims encourages players to create happy successful families, but also allows players to kill their Sims through neglect as well as indirect actions. Yet the player may be rewarded by the game for such violent actions (getting that familywrecker out of the home, for example). We should not be so quick to question such actions, if we do believe games really are a space apart, governed by a "different" set of rules.

What results when such judgments are applied is an infantilization of the game space. It suggests players cannot understand a separate set of rules and rewards, or that we can have no spaces where such alternate systems might function. A one-to-one mapping of values robs games of their unique character, and their rule set, creating a space derivative of 'real life' standards of behavior. When that happens, choices that might be interesting or significant within a game are diminished, and choices are robbed of their playful, experimental quality. And the game space becomes impoverished, leaving game players with two sets of rules to negotiate-the in-game rules for rewards, and the daily life rules that impose larger judgments onto their actions.

Where does such theorizing of play and games leave us, in relation to ethics? Obviously play and games are central parts of the human experience, and ethics are likewise centrally placed in our lives. How do the two come together? To suggest that games are a "space apart" from daily life and our normal rules for living is just as much of an ethical choice as making them conform to and integral with our daily codes of conduct. We cannot say that there are "no ethics" in games or that players bring no ethical frameworks to their gameplay-instead we leave the question unexamined, which is itself a choice. What we need to do instead is actively involve ourselves with the questions, seeking to determine how ethics fit, how we see them informing games and gameplay, and how we choose to integrate games into our lives (or not). Although not tied specifically to games or ethics, one way of beginning that discussion is through theories of active media audiences.

\section{Cheating in games and daily life}

My own work has focused on how individual players have defined and negotiated various cheating behaviors in their regular gameplay. As I have learned, many players define cheating in a fairly restrictive way and then proceed to "break the rules" with abandon. In a different context (such as writing a paper for a school assignment) such rule-breaking might be troublesome, but here something different is at play. While some players do certainly keep connections between the rules of their non-gaming and gaming lives, others draw distinctions between them. For some (if not many) players, the game world is a space apart where normal rules don't apply.

Leaving aside the question of whether a magic circle is operating or not, such behaviors raise interesting questions about the role of games in our lives. For many players, playing games is, in some measure, a playing with rules and their boundaries. Games offer a bounded space (although some games are more bounded than others, depending on how many people are playing) for the exploration of actions and consequences as well as the ludic expression of activities deemed inappropriate (if not illegal) in regular life.

Many players cheat in games (single as well as multiplayer) to "play God" or have fun, without necessarily wanting to get ahead or defeat another human player. Such individuals have made a decision that while their activity may or may not be selfdefined as cheating, such shortcuts or code alterations are acceptable in the space of the game. Huizinga reminds us that games are a "stepping out" of real life into a space apart. Although more games are now following us into real life (IMs from guildmates, phone calls to friends to enter the game 
and help out, real-money trade that alters game economies), the space of the game itself instantiates particular rules which players must negotiate. And apart from breaking the terms of a EULA, there are few "real" consequences for breaking the rules of a game.

Similarly, many players "cheat" in games when they get stuck. Having reached a point where they cannot progress further without help, they turn to guides, codes, or friends to help them get past the point of difficulty. This is the most common and accepted form of cheating (some players don't see it as cheating at all), suggesting that the reaching of an impasse and resulting request for help is something not very divorced from regular life.

Likewise, players cheat in order to "fast forward" through unpleasant or boring parts of a game, in order to reach its endpoint. That practice, found in single and multiplayer games (using cheat codes to skip levels or using a power-leveler in a MMOG) is usually instrumental in nature, recognizing that a player wishes to complete a game yet not fully engage all aspects of it. Most of the time we can't fast forward through our lives, and even if we could, we actively choose not to. Most students research and write papers rather than finding one on the Internet to download, and most drivers stop at deserted intersections, even if no police are in sight. Yet games offer us a space where we can experience that freedom, without significant consequences.

What is unfortunate is that popular discourse tends to judge in-game behaviors by the rules that operate in daily non-game life. I can see this in the way that many players have defended their actions, trying to reassure me that a particular code use was "necessary" to continued progress in a game. Players also state that "it's just a game" as a way to deflect criticism in advance of their actions. But why must we hold our actions in games to what is really a separate standard? Why don't we allow for more play and variation in games, allowing players to experiment with actions, identities, and practices that in "real life" are forbidden?

Individuals might find in games a space to explore the consequences of various actions, and challenge or reify their own beliefs about what are appropriate or inappropriate actions to take in specific circumstances. They can also play at taking what are normally the "wrong" actions for them in daily life, gaining perspective on other choices made. We expect children to play but adults are considered juvenile when engaging in "childish" actions. Games are and can become even better at becoming spaces for exploration of not only fantastical worlds and rhetorics of power, but also of playing with rules and their boundaries.

\section{"Is this a good game or a bad game?"}

When the question above is posed, typically, two groups have done the asking, and they are addressing fundamentally different issues concerning games which have more to do with judging games than examining ethical processes. Those groups are game players and game critics (such as politicians and activist groups).

When game players ask 'is this a good or bad game' they are asking whether the game will entertain them, if the story is intriguing, if the gameplay delivers what the marketing promises, and if the game is thus successful at providing entertainment value. When politicians and interest groups ask the question, it invokes issues such as the glorification of violence and the amount of violence in a particular game, whether there are prostitute or drug dealers or other criminal 'types' present, whether or not the game depicts women or minorities in a negative light, how religions (including the occult) are shown, and how all those elements might influence an impressionable child.

Both sets of questions are banal, I believe, and neither addresses the question of ethics. While game studies scholars have begun to examine gameplay to determine what it encompasses (it's definitely a slippery term), it has not been tied in any formal way to an expression of ethics. At the same time, the questions of good and bad that are raised by such groups often have little to do with better understanding games and player experiences with them-rather they are tied to calls for censorship or general moral outrage.

What this paper has outlined, in contrast, is a path to exploring more interesting questions about games and ethical choices. We can study how games are a space apart with separate rules and rewards, and we can also explore how games are spilling over into our daily lives in pleasurable and troublesome ways, with real consequences. That approach takes us further, I believe, in understanding the role of games in contemporary culture, and how we negotiate our beliefs as we play at them, either walled off or happily integrated into the rest of our lives. 


\section{References}

Fiske, J. (1987). Television culture. New York: Routledge

Goldstein, J. (2005). Violent video games. In J. Raessens and J. Goldstein (Eds.), Handbook of computer game studies, (pp. 341-358). Cambridge, MA: The MIT Press.

Hall, S. ([1973] 1980). Encoding/decoding. In Centre for Contemporary Cultural Studies (Ed.), Culture, media, language: Working papers in cultural studies, 1972-79 (pp. 128-138). London: Hutchinson.

Huizinga, J. (1955). Homo ludens: A study of the play element in culture. Boston: Beacon Press.

Jenkins, H. (1999). Professor Jenkins goes to Washington. Harper's magazine. Available online at http://web. mit.edu/cms/People/henry3/publicati ons.html

Kellner, D. (2005). Cultural studies, multiculturalism, and media culture. Accessed online at http://www. gseis. ucla.edu/faculty/kellner/

Reynolds, R. (2002). Playing a "good" game: $A$ philosophical approach to understanding the morality of games. International Game Developers Association, available online at http://www.igda.org/articles/rreynolds_ethics.ph $\underline{p .}$

Sherry, J. (2001). The effects of violent video games on aggression. Human Communication Research, 27, 409-431. 